\title{
Contact Location Trajectory on the Fingertip as a Sufficient Requisite for Illusory Perception of Haptic Shape and Effect of Multiple Contacts
}

\author{
Hanifa Dostmohamed and Vincent Hayward \\ Haptics Laboratory, Center for Intelligent Machines \\ McGill University, Montréal, QC, H3A 2A7, Canada \\ \{hanifa,hayward\}@ cim.mcgill.ca
}

\begin{abstract}
This paper seeks to demonstrate that haptic curvature perception can result solely from the trajectory of the deformed region of the fingertip due to contact with an object during tactile exploration. To test this hypothesis, we built a servo controlled 2-degree-of-freedom spherical mechanism called a Morpheotron. This device operates by rolling a flat plate on the fingertip during the exploration of a virtual surface while eliminating all other cues that are normally present, including kinesthetic cues. In carrying out the experimental protocols described herein, we found that subjects were able to perform curvature discrimination at levels comparable to those achieved when using direct manual contact with real objects, and that the highly simplified stimulus provided was able to give the illusion of touching three dimensional surfaces.
\end{abstract}

\section{Introduction}

A better understanding of how tactile cues determine the perception of object attributes has potential to yield more systematic methods for the engineering of haptic interfaces. In this paper, we examine the effect of a highly simplified haptic signal that is created when a flat plate rolls on the fingertip while an operator explores the surface of a virtual object. This is equivalent to eliminating all cues that normally are present during the interaction of a finger with an object with the sole exception being the change in location of the contact region on the finger. To display this highly simplified haptic signal, we created a programmable haptic feedback mechanism for three dimensional shape display which was very simple to implement.

Most haptic devices relay shape and curvature information through force feedback, assuming that a "virtual probe" serves as an intermediary between the stimulus and the operator's finger(s). With displays relying on this assumption, judging shape and curvature information is clearly at best as inefficient as in the real condition of being forced to explore and judge the shape of a real object through the use of a stylus $[4,1,3]$. There currently exists ample evidence that distributed finger deformation due to direct contact is critical and in fact the key to many haptic tasks [5]. 


\section{In Multi-point Interaction with Real and Virtual Objects, F. Barbagli, D. Prattichizzo and K. Salisbury (Eds.), Springer Tracts in Advanced Robotics. Volume 18, pp. 189-198.}

Other devices designed to provide a haptic experience without having to assume the existence of an intermediary probe sliding on the virtual object were described in $[15,9,8]$. None of these devices, however, is as radical as ours in their attempts to eliminate all forms of haptic feedback cues but one: that of deforming the finger tip in a manner that resembles exploring real objects. The following diagram illustrates this idea.

$\mathbf{a}$
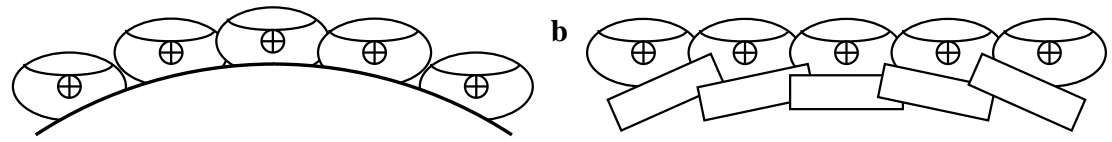

Fig. 1. Finger tip deformation as a method for relaying shape.

Fig. 1a represents a finger sliding on an object in order to feel its shape. Readers should notice, by experimenting on themselves, that although many maneuvers are possible for this task, the movements that keep the finger orientation invariant, such as in movement along the lateral direction, are common.

We built a servo-controlled mechanism, that we called a "Morpheotron", which oriented a plate around a center of rotation located inside the user's finger as it rested on the plate (as close to the bone's distal end as we could). When the plate changed orientation, it "rolled" on the finger leaving the bone nearly stationary as illustrated by Fig. 1b, thus mimicking the deforming action caused by an object on the finger and resulting in a strong sensation of shape.

In a first experiment (Experiment I), our objective was to demonstrate that shape could be correctly perceived by subjects with varying deformation of the fingertip at the contact area as the sole cue. In a second experiment (Experiment II), we sought to identify the conditions under which performance in the perception of shape was enhanced.

Earlier studies have shown that significant differences exist in judging curvature when comparing active versus passive touch [10]. We were able to test this with our device by mounting it ontop of a gantry that allowed it to be frictionlessly rolled along the $x$ and $y$ directions in a fashion similar to that of moving a mouse. When the Morpheotron was mounted ontop of the gantry, subjects explored the shape of a stationary virtual object by moving the Morpheotron with the same hand as that whose finger(s) rested on the plate experiencing the shape (we refer to this as active exploration in the context of this study). In a separate condition, the Morpheotron was immobilized by removing the gantry and subjects used their other free hand to move a computer mouse that determined the position on the virtual object while the finger(s) of the other hand experienced the shape (we refer to this as passive exploration in the context of this study). In this condition, the hand that experienced the shape was passive, however the one that explored the virtual surface was active.

Finally, we attempted to identify whether the use of multiple points of contact increased the performance in shape judgment tasks since it is not uncommon to use multiple fingers while exploring curvatures of real objects. We tested this by 
In Multi-point Interaction with Real and Virtual Objects, F. Barbagli, D. Prattichizzo and K. Salisbury (Eds.), Springer Tracts in Advanced Robotics. Volume 18, pp. 189-198.

asking subjects to engage either one or two fingers while experiencing the stimuli (in the context of this study we refer to these constraints as the use of single versus multiple points of contact respectively). The effect of using single and multiple points of contact to experience the shape was tested using both the active and passive exploration methods. The above constraints collectively resulted in the testing of subjects for performance gain in shape judgment tasks using active versus passive exploration and in the use of single versus multiple points of contact.

We found that the single cue provided by the device, the deformation of the fingerpad at the contact area, was very efficient at relaying shape in that subjects performed at a level equivalent to using direct manual contact with real objects

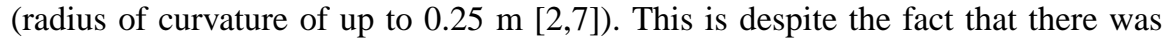
no shape information related to changes in the interaction force since this force was entirely determined by the user (i.e. the user determined the amount of force applied when exploring a surface); there was no information related to changes in traction distribution on the fingertip since the contact surface was always flat; and there was no kinesthetic cues associated to shape because the finger tip always moved in a plane or did not move at all. We were also able to show that under active exploration, the use of multiple points of contact provided a notable increase in performance of judging shape information as opposed to the use of a single point of contact. From the results, we can conclude that the perception of curved three dimensional surfaces given by only the trajectory on the fingertip of the contact region with an object is illusory.

\section{Experiments}
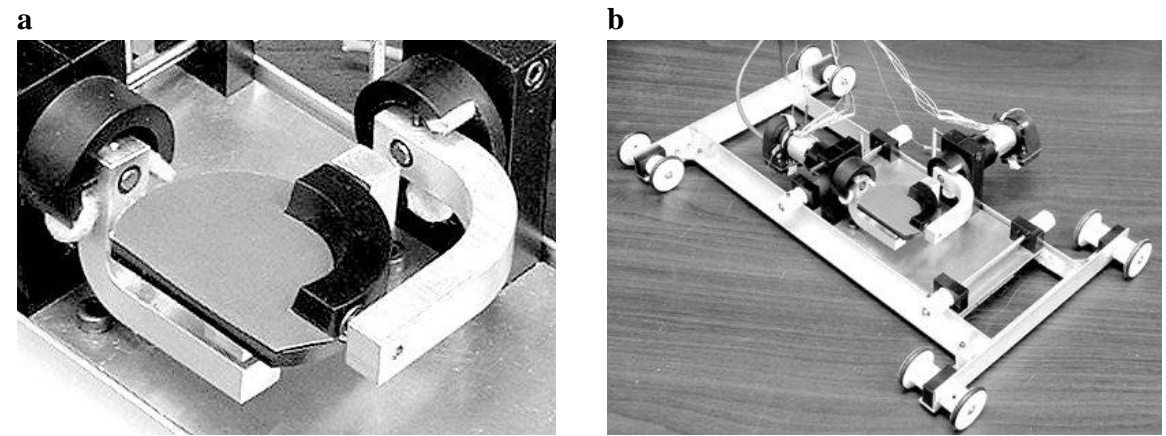

Fig. 2. a) Servo-controlled spherical linkage. b) Morpheotron on lightweight gantry.

\subsection{Apparatus}

Fig. 2a shows the construction of the Morpheotron. It was designed with a spherical five-bar linkage which is a parallel linkage such that the driven link rotates around 


\section{In Multi-point Interaction with Real and Virtual Objects, F. Barbagli, D. Prattichizzo and K. Salisbury (Eds.), Springer Tracts in Advanced Robotics. Volume 18, pp. 189-198.}

a fixed point called the center of rotation. The center of rotation was located $3 \mathrm{~mm}$ above a non-slip plate on which the finger rested. This allowed us to display threedimensional virtual surfaces directly to the finger pad. The mechanism was driven by miniature DC motors with gear-head reduction which tracked reference signals suitable to orient the plate tangentially to the virtual object being explored. The Morpheotron could be controlled either through the use of the gantry shown in Fig. 2b or without it (i.e. active exploration versus passive exploration). In either case, the position over a virtual surface was collected using an optical tracking system of a quality computer mouse (Logitech M-BJ69 800dpi Optical Wheel Mouse). When the device was mounted on top of the gantry, the optical tracking system that was mounted on the underside of the gantry was used to collected the position, however when the gantry was not used, the device was immobilized and the position was collected using the optical tracing system of a separate mouse of the same brand. In both cases, the finger(s) that was exploring the virtual stimuli remained stationary on the plate of the device while the position over the virtual stimuli was obtained using a variety of exploration methods (such as fore-aft, lateral and spiral motions) of the optical tracking system.

\subsection{Methods}

Stimulus. Virtual shapes were presented to the finger pad(s) using the device described above. For both Experiments I and II, shapes consisted of convex and concave sections of spheres. In Experiment I, curvatures that were easy to discriminate $\left(1 / r= \pm 22, \pm 18, \pm 15 \mathrm{~m}^{-1}\right)$ were presented in order to judge whether subjects were simply able to discriminate shape from the sole cue that the device provided. In Experiment II, curvatures ranged from \pm 2.5 to $\pm 10 \mathrm{~m}^{-1}$ (i.e. $1 / r= \pm 2.5$, $\left.\pm 2.85, \pm 3.33, \pm 4.00, \pm 4.50, \pm 5.00, \pm 5.50, \pm 6.67, \pm 8.33, \pm 10.00 \mathrm{~m}^{-1}\right)$ in order to bracket the discrimination threshold (spheres of radii from 0.4 to $0.1 \mathrm{~m}$ ). To maximize data and minimize the time required from participants, the number of presented stimuli for each curvature category varied in Experiment II. Curvatures which from Experiment I were observed to be easier to discriminate (i.e. curvatures $>5.5 \mathrm{~m}^{-1}$ ) were presented less often than those that were more difficult to discriminate. In the conditions where the subjects actively explored surfaces, the apparatus permitted movement over a $280 \times 280 \mathrm{~mm}$ range, however shape information was only displayed within a $50 \times 50 \mathrm{~mm}$ window and the plate was brought to rest if the exploration exceeded the boundary of the window. The range was limited for all conditions and for both Experiments I and II. Previous studies have shown that the dimension of a stimulus can directly influence the judgment of haptic curvature discrimination [6]. The range was therefore limited for two reasons: (1) To limit subjective bias that is introduced with increasing stimulus length and (2) to limit the amount of proprioceptive cues that resulted from the movement of the finger when the device was mounted on top of the gantry during active exploration. 
In Multi-point Interaction with Real and Virtual Objects, F. Barbagli, D. Prattichizzo and K. Salisbury (Eds.), Springer Tracts in Advanced Robotics. Volume 18, pp. 189-198.

Subjects. The first experiment was conducted with four right-handed subjects (A, B, C, D) (two male and two female in their twenties). Two of the subjects were naive as to the purpose and design of the device, and two knew of it from working in the same laboratory as the authors, but did not know of the experimental protocol beforehand. The second experiment was carried out with four right-handed subjects (D, E, F, G) (one female and three male in their twenties). Three of the subjects were naive as to the purpose and design of the experiment and one knew of the device. Subject D participated in all experiments. The subjects were compensated for their participation.

Procedure. Under all experimental conditions, subjects were seated with their forearm resting on the table passing under a curtain so they could not see their hands. There was a brief training session which consisted of 12 trials where subjects were instructed to experience virtual shapes in the same conditions as in the experimental test trials but were aware of the profile of the curvature and the size of the sphere being presented. During the experimental trials, the virtual spherical shapes were randomly presented. Subjects submitted their responses by pressing keys labeled convex and concave using their left hand. Subjects were timed as to how long it took them to enter a response. No time limit was imposed but subjects were simply asked to enter their answers as soon as they were ready to make a choice. No feedback was given to subjects during the experimental phases. The four conditions of Experiment II are illustrated in Figure 3.

a

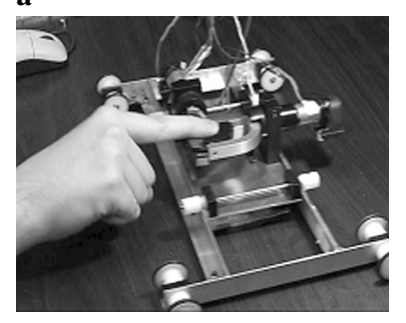

c

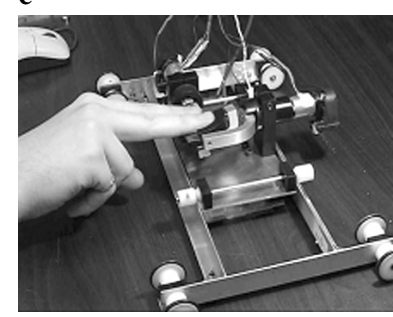

b

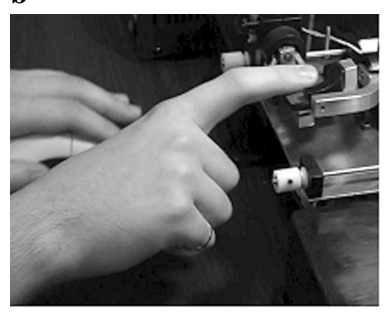

d

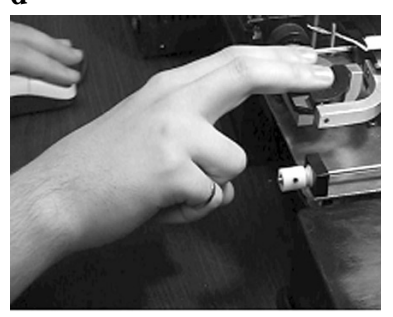

Fig. 3. The four conditions that were employed in Experiment II: a) Active exploration using a single point of contact $\mathbf{b}$ ) Passive exploration using a single point of contact $\mathbf{c}$ ) Active exploration using multiple points of contact d) Passive exploration using multiple points of contact. 
In Multi-point Interaction with Real and Virtual Objects, F. Barbagli, D. Prattichizzo and K. Salisbury (Eds.), Springer Tracts in Advanced Robotics. Volume 18, pp. 189-198.

Data collection. For Experiment I, the responses of each subject (i.e. whether the stimuli presented was judged to be convex/concave) were recorded and the percentage of correct answers tabulated. In Experiment II we used a variation of a two alternative forced choice (2-AFC) paradigm in which one stimulus (instead of two) was presented and the subject was required to choose from one of two possibilities: convex or concave. We calculated $p$ (correct), the probability of classifying stimuli with varying curvature correctly as convex or concave for each subject under each of the four conditions. The probabilities were averaged for each independent stimuli since the number of stimuli presented varied across curvatures as was described above. The data for each subject were then fit with a Weibull function in order to determine the thresholds of curvature detection at $60 \%, 75 \%$ and $90 \%$ levels. Psychometric function parameters were obtained using maximum likelihood estimation and subsequent Monte Carlo simulations in order to assess the goodness of fit. These analyses were largely carried out using SPSS ${ }^{\mathrm{TM}}$ and the psignifit toolbox [13,14]. A representative example of how thresholds were obtained from fitted psychometric functions is illustrated in Fig. 4.

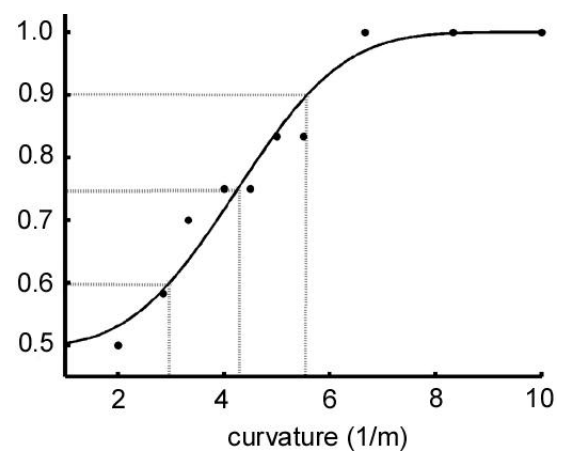

Fig. 4. Representative example of the Weibull psychometric function fitted to data points representing a subject's performance with varying curvature under a specified condition. The ordinate shows the proportion of correct responses in categorizing curvatures as convex or concave. The abscissa shows the curvatures that were randomly presented.

\section{Results}

Basic Curvature Discrimination. The results of Experiment I are summarized in Fig. 5. All of the subjects had a performance at or near $100 \%$.

Effect of Dual Contact Points During Active and Passive Exploration. The performance of subjects under all conditions in Experiment II is summarized in Table 1. In order to test whether multiple points of contact during active exploration of virtual shapes had an effect on performance, we carried out a two factor without 
In Multi-point Interaction with Real and Virtual Objects, F. Barbagli, D. Prattichizzo and K. Salisbury (Eds.), Springer Tracts in Advanced Robotics. Volume 18, pp. 189-198.

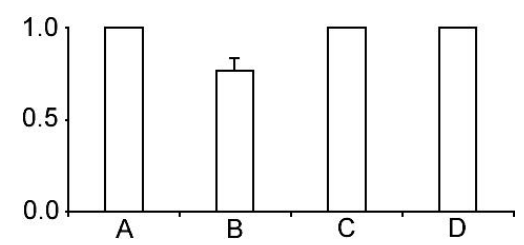

Fig. 5. Results showing performance of subjects during Experiment I.

replication analysis of variance test (ANOVA). A significant difference was observed between the two groups indicating that the number of contact points used to explore a curved surface has an effect on overall performance during active exploration $[F(1,9)=17.18, p<0.05]$. A significant difference, however, was not found when comparing the two groups, during passive exploration $[F(1,9)=1.26, p>0.05]$.

Active and Passive Exploration. To examine if there was a significant difference between active and passive exploration of curvature, single point contact exploration was compared under both conditions using a two factor without replication ANOVA test. The results show that there is a significant effect on performance due to the exploration technique used $[F(1,9)=47.68, p<0.05]$. When multiple points of contact were compared under both exploration modalities, we also found that the result was highly significant $[F(1,9)=36.43, p<0.05]$.

Table 1. Performance (in $\mathrm{m}^{-1}$ ) at $\mathrm{T}_{75}$ and average time (in seconds) for each subject under all conditions.

\begin{tabular}{lcccccccc}
\hline & \multicolumn{2}{c}{$\begin{array}{c}\text { active } \\
\text { two finger }\end{array}$} & \multicolumn{2}{c}{$\begin{array}{c}\text { active } \\
\text { one finger }\end{array}$} & \multicolumn{2}{c}{$\begin{array}{c}\text { passive } \\
\text { two finger }\end{array}$} & \multicolumn{2}{c}{$\begin{array}{c}\text { passive } \\
\text { one finger }\end{array}$} \\
\hline Subject & $\mathrm{T}_{75}$ & Time & $\mathrm{T}_{75}$ & Time & $\mathrm{T}_{75}$ & Time & $\mathrm{T}_{75}$ & Time \\
\hline $\mathrm{D}$ & 4.02 & 10 & 3.96 & 9 & 4.29 & 12 & 4.74 & 12 \\
$\mathrm{E}$ & 3.33 & 12 & 3.83 & 16 & 5.38 & 15 & 3.96 & 10 \\
$\mathrm{~F}$ & 4.26 & 13 & 5.35 & 11 & 5.81 & 10 & 6.71 & 13 \\
$\mathrm{G}$ & 4.72 & 8 & 5.01 & 9 & 5.52 & 13 & 6.71 & 10 \\
Av. & & 11 & & 11 & & 13 & & 12 \\
Av. $^{\text {a }}$ & 4.08 & & 4.54 & & 5.25 & & 5.53 & \\
Av. $^{\text {b }}$ & 3.96 & & 4.40 & & 5.38 & & 5.29 & \\
\hline
\end{tabular}

${ }^{\text {a }}$ Thresholds from simple average of $\mathrm{T}_{75}$ values for each subject across each condition.

${ }^{\mathrm{b}}$ Thresholds from Weibull function fit to data obtained from averaging individual probabilities across subjects and conditions. 
In Multi-point Interaction with Real and Virtual Objects, F. Barbagli, D. Prattichizzo and K. Salisbury (Eds.), Springer Tracts in Advanced Robotics. Volume 18, pp. 189-198.

Recognition Time and Accuracy. As summarized in Fig. 6a, the time required to judge a stimulus as concave or convex was observed to be the smallest with active exploration using multiple contact points. It was the largest during passive exploration with multiple points of contact. A trend could be observed in the threshold at which subjects were able to correctly identify $75 \%$ of the stimuli presented. On average, subjects could identify smaller curvatures when actively exploring the stimuli using multiple points of contact. The threshold of detection decreased with one contact points during active exploration. With passive exploration, the threshold increased and using multiple points of contact was found to provide a slight advantage over the single point of contact. The percentage correct for each modality was calculated and averaged across all subjects. The mean score for participant accuracy are presented in Fig. $6 b$.

a

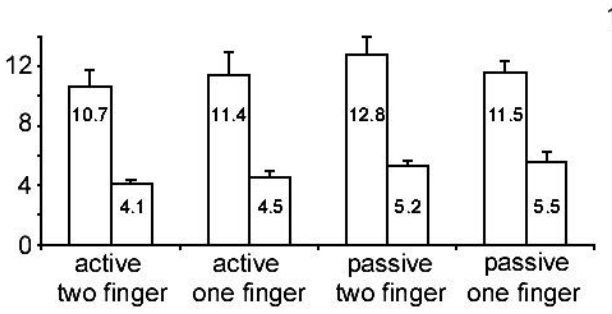

b

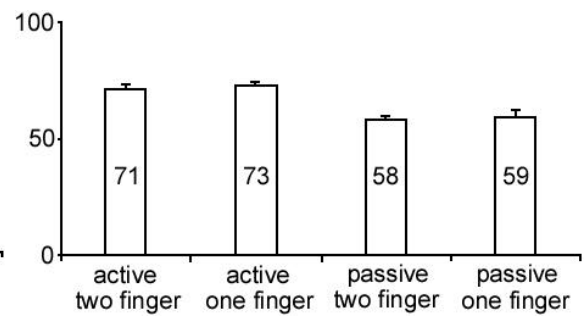

Fig. 6. a) Recognition time (seconds, left bars) and threshold values $\left(\mathrm{m}^{-1}\right.$, right bars) at $p=0.75$ level averaged over all subjects for all conditions. b) Mean score for subject accuracy in all conditions ( $\mathrm{s} . \mathrm{d}=3.56,2.94,3.59,6.95)$.

Summary of Results. For Experiment I, the results indicate that subjects were able to discriminate between convex and concave stimuli at high curvatures with performance rates at or near $100 \%$. Figure 7 shows the Weibull curves fit to the average data obtained from all subjects under the four conditions of Experiment II. The thresholds at which subjects were able to correctly identify $75 \%$ of the stimuli were found to be $\mathrm{T}_{75}=3.96$ when using two fingers actively, $\mathrm{T}_{75}=4.40$ using one finger actively, $\mathrm{T}_{75}=5.38$ for using two fingers passively and $\mathrm{T}_{75}=5.29$ for using one finger passively.

\section{Discussion and Conclusions}

We have shown that curvature can be perceived by a single cue which is that of deformation trajectory of the fingertip. Actively exploring a surface was shown to provide a significant advantage on performance over passive exploration of the same stimuli. A significant advantage was also observed between active explorations of stimuli using multiple points of contact as opposed to single points in that the 
In Multi-point Interaction with Real and Virtual Objects, F. Barbagli, D. Prattichizzo and K. Salisbury (Eds.), Springer Tracts in Advanced Robotics. Volume 18, pp. 189-198.

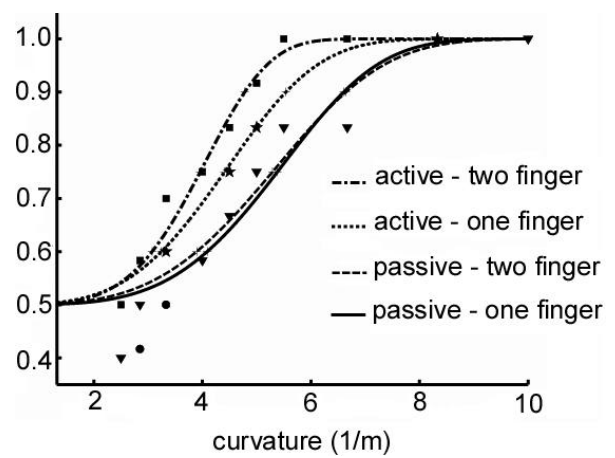

Fig. 7. Average data obtained from subjects in the four conditions of Experiment II.

threshold performance for identifying smaller curvatures correctly as convex or concave was observed to increase. We did not find any significant differences between using single and multiple points of contact when curvatures were explored using passive touch. We also did not find any significant differences in the amount of time needed to arrive at judgments across the four different conditions, however, the time required to arrive at a judgment was observed to be shorter than reported in similar studies using haptic devices for shape perception [3].

Curvature perception has previously been studied by a number of groups by utilizing real objects $[11,2,7,10]$. In comparing our results to these studies we found that using our device, subjects are able to discriminate convex and concave stimuli at a performance level comparable to those found when exploring a real object. Goodwin et al. reported that subjects were able to discriminate curvatures of $+4.9 \mathrm{~m}^{-1}$ and $5.4 \mathrm{~m}^{-1}$ at $75 \%$ correct when statically experienced [2]. Although we did not analyze convex and concave stimuli separately, we obtained similar results during passive exploration using our device. Pont et al. found that the results for static and dynamic curvature comparison in the range of $-4 \mathrm{~m}^{-1}$ to $+4 \mathrm{~m}^{-1}$ are the same [7], however in our study we show that they are quantitatively different. These differences may be accounted for by the fact that certain cues such as friction and speed of exploration are variable between the exploration of physical and virtual stimuli. We would like to investigate this possibility as a source of error in future work. Other studies have previously reported that cutaneous information and in particular compression of the finger is a major contributing factor in discrimination if two-dimensional angles $[12,5]$. They add that proprioceptive cues are important in increasing performance. In future work, we would like to incorporate these proprioceptive cues into the design of our device so as to increase its utility as a haptic interface for exploring virtual environments. Using our device, we have not yet tested for the the threshold at which objects with large curvatures can be perceived at a 75\% level accuracy as was done for stimuli with low curvatures. We suspect that the conditions which would enhance subjective performance using smaller stimuli would be quite different from those conditions which were seen to be optimal for the perception of stimuli with small curvatures. We would like to investigate these questions in future work as well. 


\section{In Multi-point Interaction with Real and Virtual Objects, F. Barbagli, D. Prattichizzo and K. Salisbury (Eds.), Springer Tracts in Advanced Robotics. Volume 18, pp. 189-198.}

\section{Acknowledgments}

This research was supported IRIS, the Institute for Robotics and Intelligent Systems, and NSERC Natural Sciences and Engineering Research Council of Canada.

\section{References}

1. C. Christou and A. M. Wing. Friction and curvature judgement. In Proc. Eurohaptics, University of Birmingham, UK., 2001.

2. A.W. Goodwin, K.T. John, and A.H. Marceglia. Tactile discrimination of curvature by humans using only cutaneous information from the fingerpads. Exp. Brain Res., 86:663-672, 1991.

3. A. E. Kirkpatrick and S. A. Douglas. A shape recognition benchmark for evaluating usability of a haptic environment. In S. Brewster and R. Murray-Smith, editors, Proc. First International Workshop on Haptic Human-Computer Interaction, pages 151-156. Berlin:Springer Verlag, 2002.

4. S.J. Lederman and R.L. Klatzky. Sensing and displaying spatially distributed fingertip forces in haptic interfaces for teleoperator and virtual environment systems. Presence: Teleoperators and Virtual Environments, 8(1):86-103, 1999.

5. W.J. Peine and R.D. Howe. Do humans sense finger deformation or distributed pressure to detect lumps in soft tissue? In Proc. ASME Dyn. Sys. and Control Div., volume DSC-64, pages 273-278, Anaheim, California, 1998.

6. S.C. Pont, A.M.L. Kappers, and J.J. Koenderink. The influence of stimulus length on static haptic curvature discrimination. In G.J.F. Smets A.M.L. Kappers, C.J. Overbeeke and P.J. Stappers, editors, In: Studies in Ecological Psychology, pages 69-72. Delft University Press, The Netherlands, 1996.

7. S.C. Pont, A.M.L. Kappers, and J.J. Koenderink. Similar mechanisms underlie curvature comparison by static and dynamic touch. Perception and Psychopysics, 61(5):874-894, 1999.

8. W.R. Provancher, K.J. Kuchenbecker, G. Niemeyer, and M.R. Cutkosky. Perception of curvature and object motion via contact location feedback. In P. Dario and R. Chatila, editors, Reprints of the 11th International Symposium on Robotics Research, 2003.

9. M.A. Salada, J.E. Colgate, M.V. Lee, and P.M. Vishton. Fingertip haptics: A novel direction in haptic display. In Proc. 8th Mechatronics Forum International Conference, University of Twente, Enschede, Netherlands, 2002.

10. I.M.L.C Vogels, A.M.L. Kappers, and J.J. Koenderink. Influence of shape on haptic curvature perception. Acta Psychologica, 100:267-289, 1999.

11. J. Voisin, G. Benoit, and C.E. Chapman. Haptic discrimination of object shape in humans: Two-dimensional (2-d) angle discrimination. Exp. Brain Res., 145:239-250, 2002.

12. J. Voisin and C.E. Chapman. Haptic discrimination of object shape in humans: Contribution of cutaneous and proprioceptive inputs. Exp Brain Res., 145(2):251-260, 2002.

13. F.A. Wichmann and N.J. Hill. The psychometric function i: Fitting, sampling and goodness of fit. Perception and Psychophysics, 63(8):1293-1313, 2001.

14. F.A. Wichmann and N.J. Hill. The psychometric function ii: Fitting, bootstrap based confidence intervals and sampling. Perception and Psychophysics, 63(8):1314-1329, 2001.

15. T. Yoshikawa and A. Nagura. A touch and force display system for haptic interface. In Proc. IEEE Int. Conf. Robotics and Automation, pages 3018-3024, 1997. 